\title{
Velocity control of nanoliter droplets using a pneumatic dispensing system
}

\author{
Sangmin Lee, In Ho Choi, Young Kwon Kim and Joonwon Kim
}

\begin{abstract}
This paper introduces a pneumatic dispensing system to control the velocity of nanoliter droplets with small variation of volume. The system consists of a flexible membrane integrated with a backflow stopper. This unique dispensing mechanism can control the velocity of droplets according to applied positive pressures regardless of other operating conditions and design parameters. The range of droplet velocities is shifted by the flow resistance at the outlet under the same cross-section area. Our dispensing system can eject droplets of desired volume at a velocity that can be easily controlled by selecting design parameters and operating conditions. This dispensing system will provide a reliable performance within an optimized condition stably to deposit droplets onto accurate locations.
\end{abstract}

Keywords: Pneumatic dispenser; Droplet velocity; Directionality; Flow resistance

\section{Introduction}

Microdispensing systems (e.g., inkjet printing system), which can precisely deposit small volumes of functional materials, provide a low cost process in aspect of saving materials and a flexible method because of forming micropatterns directly without masks for patterning [1-5]. The dispensing systems have been widely applied as a deposition and patterning tool in various manufacturing processes in the electronics and micro-engineering industries. Examples include patterning electrodes, soldering electric circuits, fabricating multicolor polymer light-emitting diodes or liquid-crystal display color filters, and depositing UV-curable resin [6-11]. Also, the dispensing system has been adapted to biological and tissue engineering applications, such as producing microarrays with biomolecules and cellular structures including scaffolds [12-15].

In these applications, volume and velocity of droplets ejected by the dispensing systems substantially influence pattern resolutions during manufacturing processes [16]. Especially, to ensure highly accurate positioning of droplets in well-defined substrate locations, the velocity and direction of the droplets ejected from the outlet must be uniform [17]. The velocity of droplets affects how the droplet contacts the substrate (impact process) and how

\footnotetext{
* Correspondence: joonwon@postech.ac.kr

Department of Mechanical Engineering Pohang, University of Science and Technology, San 31, Pohang, Kyungbuk 790-784, Republic of Korea
}

straightly it flies to the substrate (directionality) [18]. High velocity provides a good directionality of the dispensed droplet but causes droplet splashing during impact process, whereas low velocity induces poor directionality and reduces the precision of positioning [19]; therefore, if a low-velocity droplet is to reach a desired location on the substrate, the droplet's flight distance must be short.

For a patterning process to be successful, the velocity of droplets must be controlled, to prevent them from splashing and to achieve accurate and precise directionality. In the most dispensing systems, a droplet's volume and velocity are both linearly dependent on the magnitude of the driving force or on the transferred kinetic energy [20-23]. Therefore, in conventional dispensing systems the droplet's velocity cannot be controlled without affecting its volume.

In this research, we introduce a pneumatic dispensing system to control the velocity of nanoliter droplets with causing small variation in their volume. The system has a flexible membrane which is deflected by applied pressure and concurrently restricted to a designed volume of a dispenser. Because of a dispensing mechanism, the volume and velocity of droplets are regulated by the volume and speed of a membrane deflection which is determined by design parameters and operating conditions. To verify relations between design parameters and the velocity of droplets, we systematically measured volume 
and velocity of droplets using various designed dispensers. We also measured how the velocity of droplets is affected by the time over which the driving force is applied and the flow resistance at the outlet.

\section{Experimental setup}

Figure 1(a) shows schematics of the pneumatic dispensing system. The dispensing system, which integrates the dispenser and reservoir, is actuated by pressures applied to the dispenser. A solenoid valve switches the pressure applied to the dispenser to be either positive or negative.

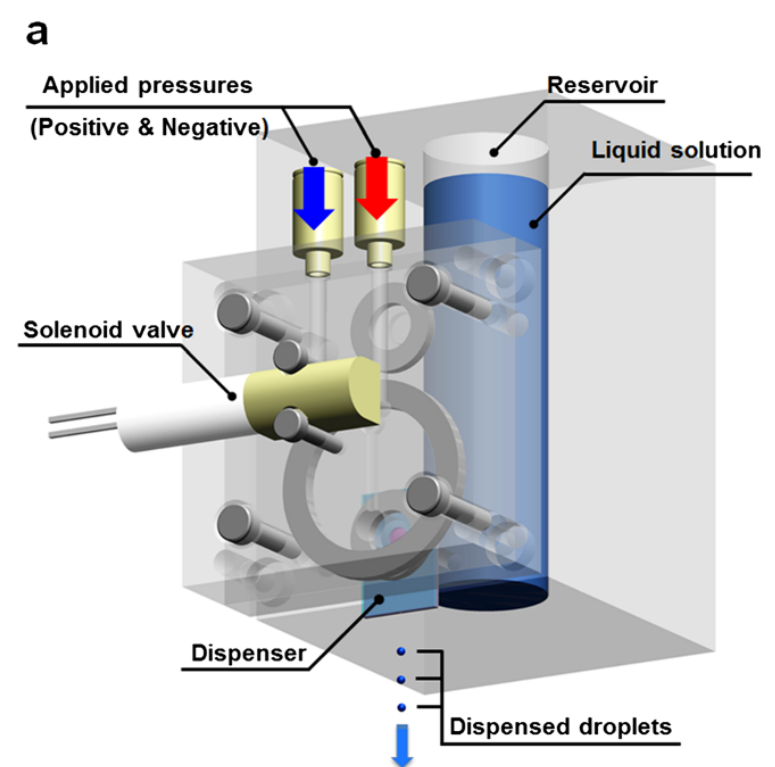

b

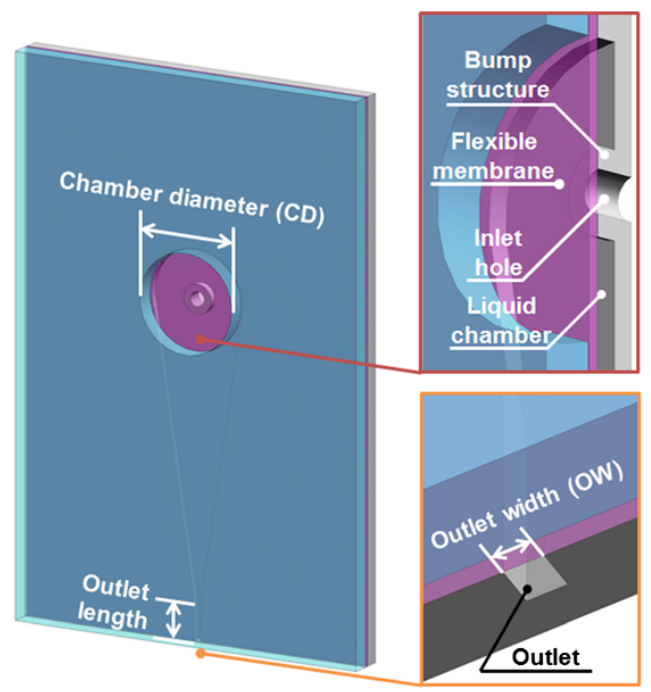

Figure 1 Schematics of a pneumatic dispensing system: (a) overview of the system and (b) design of the dispenser.
Solution delivered from the reservoir is dispensed according to a programmed signal that determines the on/ off time of the solenoid valve.

The dispenser has top (glass) and bottom (silicon) substrates separated by a flexible membrane (PDMS film). The bottom substrate includes a liquid chamber, an inlet hole, and a bump structure that functions as a restrictor during the dispensing process (Figure 1(b)). The outlet of the dispenser is oriented sideways and is defined by a dicing process of the assembled substrates. The flexible membrane is deflected by the applied pressure, and thus draws in or dispenses liquid. The membrane is either pulled (negative pressure) or pushed (positive pressure) depending on the programmed electric signal. The applied pressure is normally negative; a pulsed signal switches a solenoid valve to provide positive pressure (during duration time) to dispense the liquid. The liquid is drawn into the chamber when the membrane is pulled (during delay time) and dispensed when it is pushed [24].

Figure 2 illustrates our experimental setup to analysis volume and velocity of dispensed droplets. The droplet volume was estimated by measuring total weight differences using a precision balance. To estimate the droplet volume and prevent errors due to evaporation after dispensing, the dispensed droplets were collected inside silicone oil. For each parameter value, the difference in the total weight of the oil bath before and after the dispensing was computed and divided by the number of droplets to obtain the corresponding droplet volume. Repeated measurements using various numbers of droplets were conducted for the individual parameters. Deionized water was used for the experiments; viscosity, density, and surface tension of the material are $1.00 \mathrm{mPa} \cdot \mathrm{s}$, $998.21 \mathrm{~kg} / \mathrm{m}^{3}, 72.75 \mathrm{mN} / \mathrm{m}$, respectively [25].

To estimate the velocity of the droplet ejected from the outlet, the emerging liquid jet or droplet was recorded using a high-speed camera (REDLAKE, MotionXtra N-4) at 10,000 frames per second. By image processing using MATLAB $^{\oplus}$, a velocity profile of the liquid jet was obtained for about $13 \mathrm{~mm}$ away from the outlet. Figure 3 shows images which have same time delay $1 \mathrm{~ms}$ after the liquid emerged from the outlet at positive pressures from $20 \mathrm{kPa}$ to $200 \mathrm{kPa}$.

To characterize the relationship between design parameters and the droplet velocity, dispensers were prepared that had different dimensions: chamber diameter (CD) ranged from $2 \mathrm{~mm}$ to $3 \mathrm{~mm}$, and outlet width (OW) from $50 \mu \mathrm{m}$ to $150 \mu \mathrm{m}$. Other design parameters of the dispenser were fixed: $100-\mu \mathrm{m}$ height of chamber and outlet, $500-\mu \mathrm{m}$ diameter of inlet hole, and $1000-\mu \mathrm{m}$ diameter of bump structure. To reduce flow resistance of the outlet, we also prepared a dispenser with short outlet length which is defined by a dicing process. 


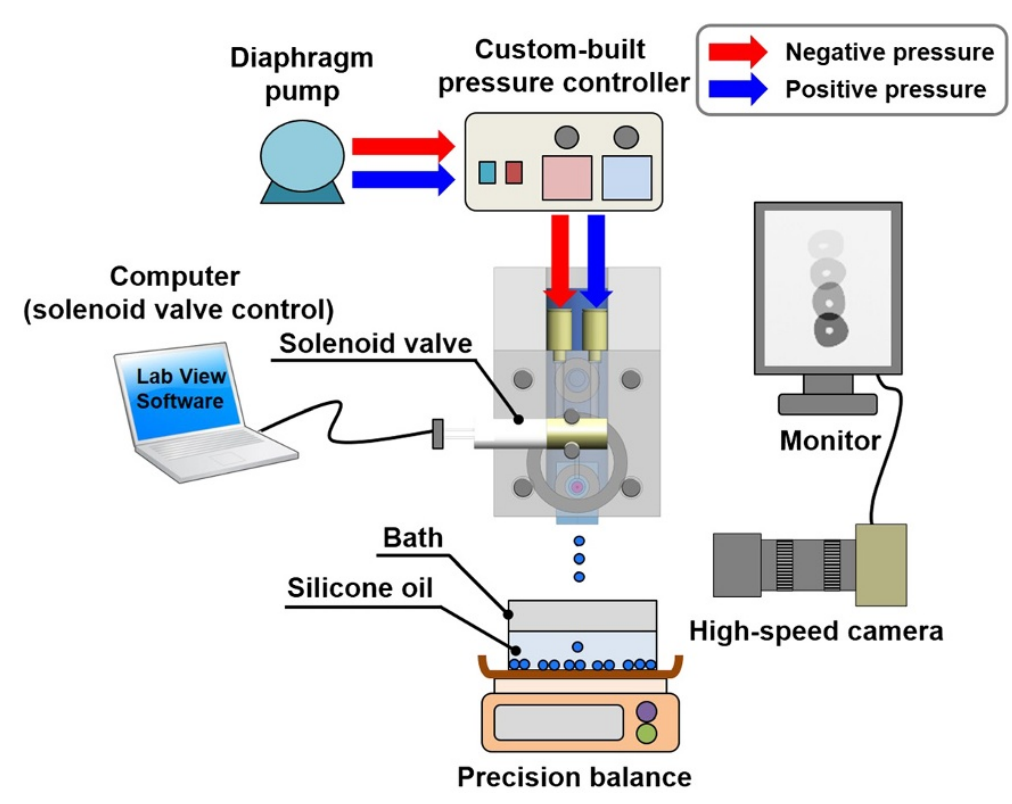

Figure 2 A schematic diagram of the experimental setup to measure the volume and velocity of dispensed droplets.

\section{Findings}

Figure 4(a) shows velocity profiles of the dispensed liquid jets or droplets for various positive pressures. The droplet was uniformly accelerated by constantly applied positive pressure, and the droplet had maximum velocity when passed through final point of experimentally observed region. The velocity of the droplet was represented by the maximum velocity in this paper. The acceleration of the droplet was proportional to positive pressure. Maximum velocity $(v)$ had relationship with positive pressure $(p)$ as follows: $v=A p^{0.4}$ (Figure 4(b)). Here, coefficient $A$ was empirically determined for each dispenser. Velocity of the droplet could be estimated using the relationship between velocity and positive pressure (i.e., controllable operating condition).

\section{A. Design parameters of the dispenser}

To determine the effect of design parameters (CD and $\mathrm{OW}$ ), droplet volume and velocity were measured for each design parameter. Positive pressures from $20 \mathrm{kPa}$ to $200 \mathrm{kPa}$ were applied while other operating conditions were fixed: 10 -ms duration time, $-10-\mathrm{kPa}$ negative pressure, $100-\mathrm{ms}$ delay time, and $1-\mathrm{kPa}$ inlet pressure.

The measured droplet volume (mean value) and standard deviation (SD) are plotted in Figure 5(a). Droplet volume increased linearly with positive pressure until volume saturation. The saturated volumes of droplets from dispensers with different chamber diameters increased from $265 \mathrm{~nL}$ to $985 \mathrm{~nL}$ because the maximum volume of that can be dispensed by the deflected membrane limited by the chamber size.

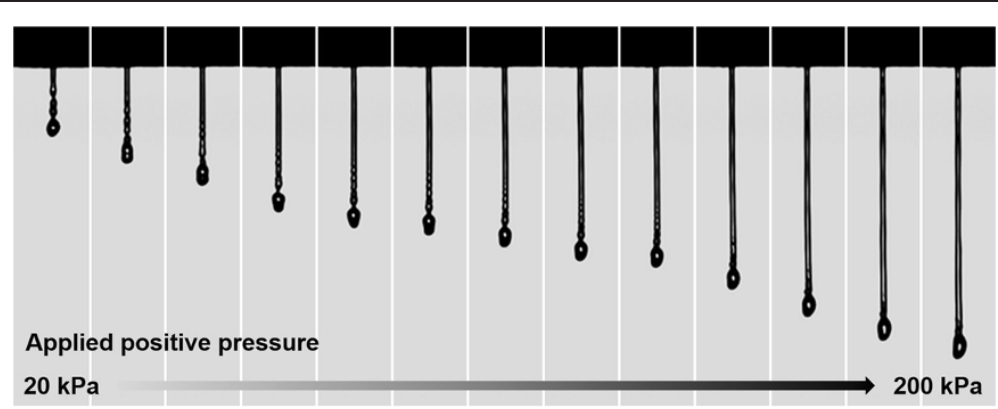

Figure 3 Liquid jets emerging from the outlet with various positive pressures after $1 \mathrm{~ms}$. 


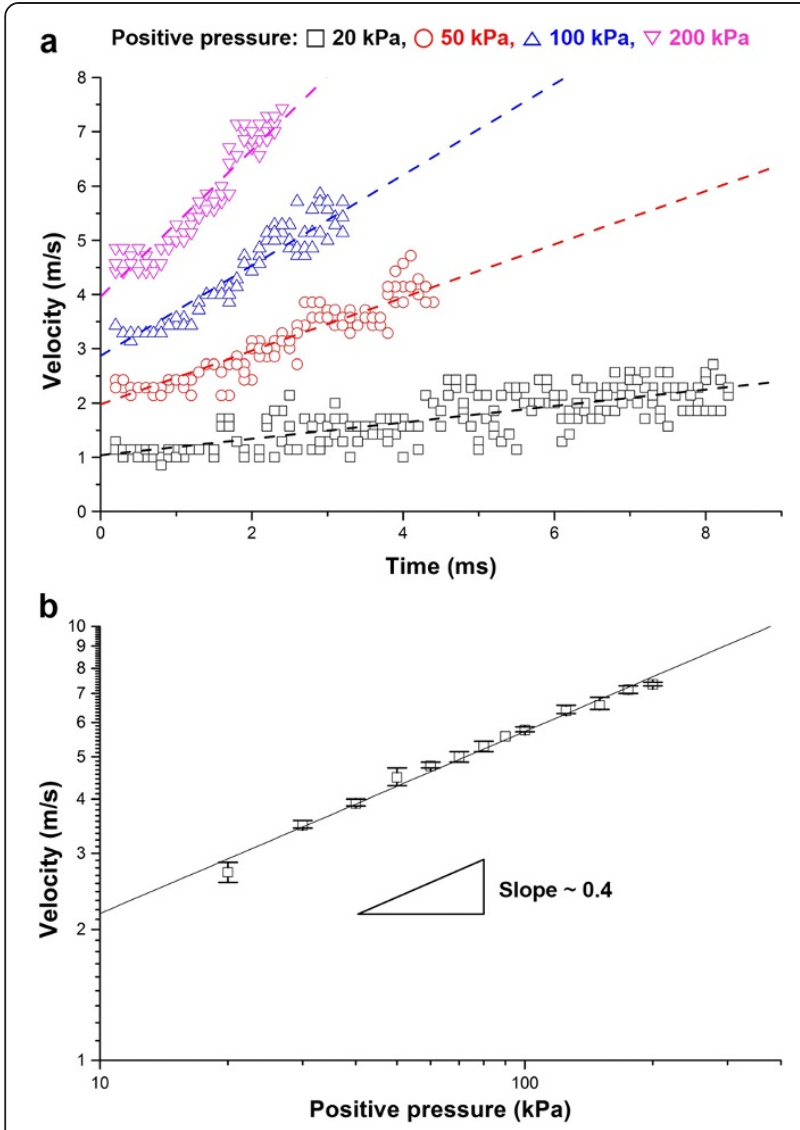

Figure 4 Relationship between velocity of the dispensed droplet and applied positive pressure: (a) velocity profiles of the dispensed droplets (dash lines are linear fitting curves for each applied positive pressure) and (b) The relationship between velocity and positive pressure (velocities were exponentially increased to positive pressure).

The volume of the droplet dispensed through the outlet was also affected by the flow resistance, which is determined by the outlet size. For outlet width-controlled dispensers at 3-mm CD, the slopes of the plot of droplet volume versus pressure increased with OW (Figure 5(a)) because the flow resistance of the outlet decreases as OW increases. The theoretically calculated flow resistances at $100-\mu \mathrm{m}$ and $50-\mu \mathrm{m} \mathrm{OW}$ are 2 times and 10 times, respectively, larger than that at $150-\mu \mathrm{m}$ OW [26]. Therefore, due to high flow resistance of the outlet, the dispenser with small OW $(50 \mu \mathrm{m})$ did not reach the saturated volume even at the maximum applied pressure.

Under the same operating condition, velocities of the droplets proportionally increased to about 0.4 th power of positive pressure before and after the volume saturation (Figure 5(b)), unlike the trend in droplet volume with pressure. For all design parameters velocities was varied from $1.6 \mathrm{~m} / \mathrm{s}$ to $8.0 \mathrm{~m} / \mathrm{s}$ while applied pressures were changed from $20 \mathrm{kPa}$ to $200 \mathrm{kPa}$. The difference between maximum and minimum velocity at the same positive pressure for

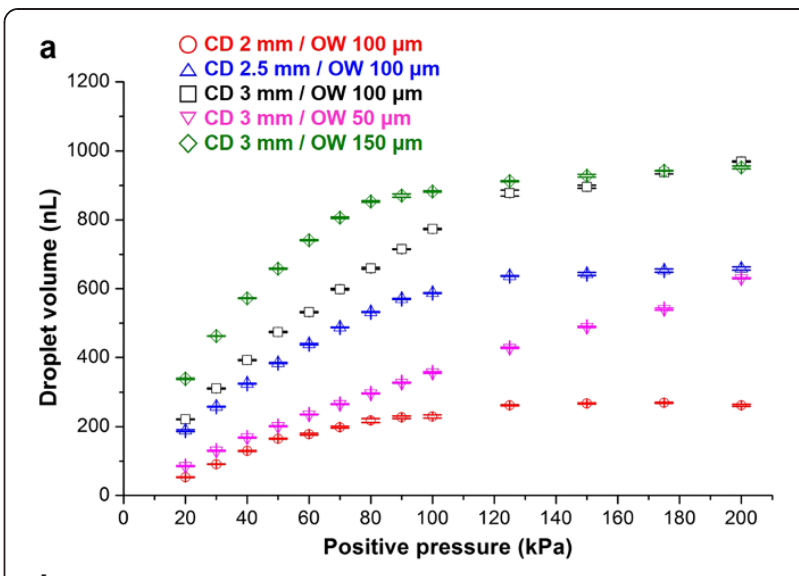

b

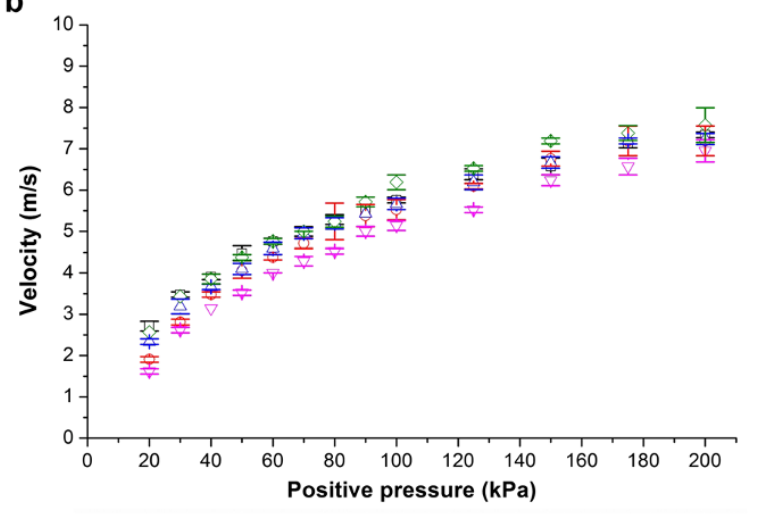

C

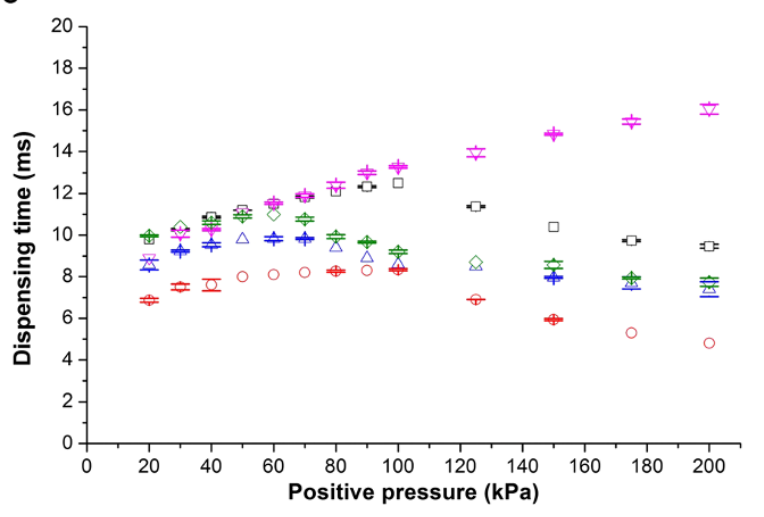

Figure 5 Characterizations of the dispensing system with various design parameters: plots of (a) droplet volume, (b) droplet velocity, and (c) dispensing time vs. positive pressure.

each design parameter was ranged from $0.9 \mathrm{~m} / \mathrm{s}$ to $1.4 \mathrm{~m} / \mathrm{s}$. (Unless the result of the $50-\mu \mathrm{m}$ OW was included, it was ranged from $0.6 \mathrm{~m} / \mathrm{s}$ to $1.1 \mathrm{~m} / \mathrm{s}$.) The velocity of droplets was dominantly affected by applied positive pressure values rather than design parameters such as CD and OW.

The velocity of droplets continuously increased over the applied pressure range even while the volume of droplets was saturated when pressure was about 100 $\mathrm{kPa}$. The dispensing time from emergence of the liquid jet to detachment at the outlet was measured from 
previously-obtained images (Figure 5(c)). Although the duration time (i.e., solenoid valve opening time) was programmed to be $10 \mathrm{~ms}$, the dispensing time changed according to dispensing condition, which determines the volume and velocity of droplets. The dispensing time tended to decrease after volume saturation to satisfy the continuity condition based on conservation of mass, because the droplet velocity increases with positive pressures. The dispensing time of the dispenser with $50-\mu \mathrm{m}$ OW constantly increased with the droplet velocity because the droplet volume does not saturated within applied pressures $(20 \sim 200 \mathrm{kPa})$.

\section{B. Duration time of application of positive pressure}

In our dispensing mechanism, the amount of input energy transferred to the liquid in the dispenser chamber is determined by the magnitude of positive pressure to the membrane and the duration time over which the pressure is applied. The dispensed volume corresponds to amount of input energy induced by membrane deflection, but the velocity of droplets increased with the magnitude of positive pressure. To determine how input energy affects the dispensed droplet, we measured the

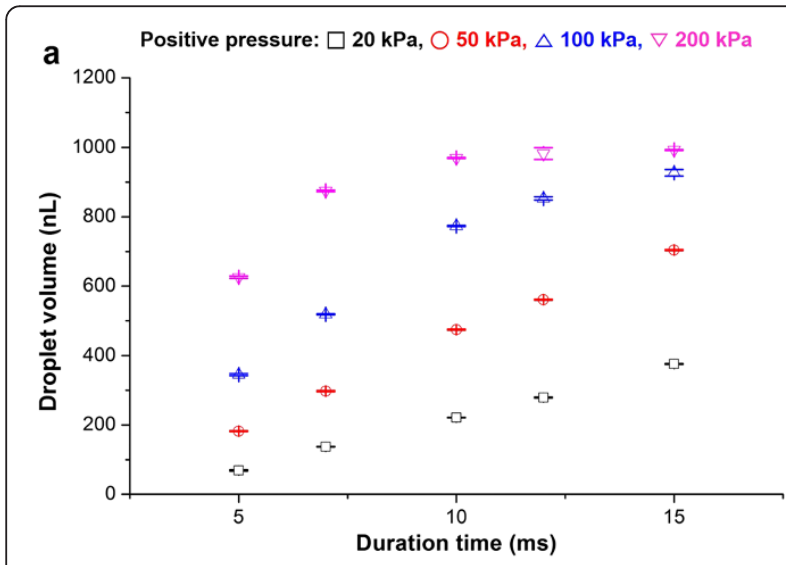

b

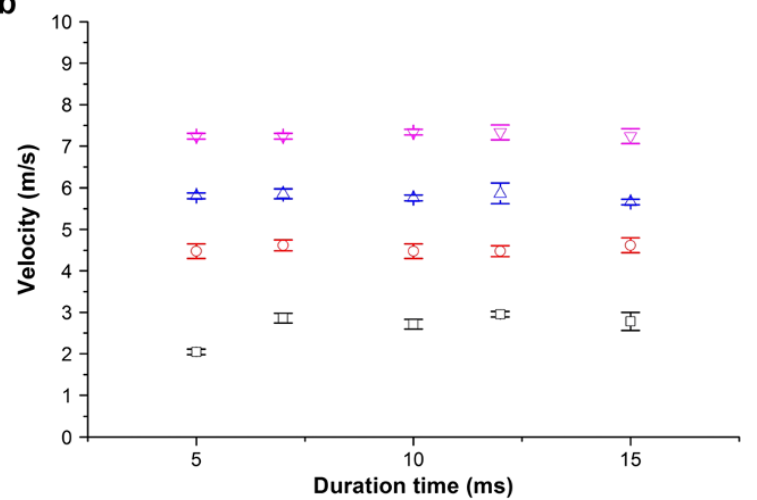

Figure 6 Effect of time to apply positive pressure (duration time) on the volume and velocity of droplets: plots of (a) droplet volume and (b) velocity vs. duration time. volume and velocity with controlled time to apply positive pressure (i.e., driving force).

Figure 6 shows the measured droplet volume and velocity at duration time from $5 \mathrm{~ms}$ to $15 \mathrm{~ms}$ with various positive pressures from $20 \mathrm{kPa}$ to $200 \mathrm{kPa}$ using a dispenser with CD $3 \mathrm{~mm}$ and OW $100 \mu \mathrm{m}$. Droplet volumes at each positive pressure increased from $310 \mathrm{~nL}$ to $580 \mathrm{~nL}$ as duration time increased the amount of input energy (Figure 6(a)). On the other hand, the droplet velocity remained consistent (variation $<0.5 \mathrm{~m} / \mathrm{s}$ ) at each applied pressure regardless of duration time (Figure 6(b)). Therefore, when the amount of input energy was varied by controlling duration time over which constant positive pressure applied, droplet velocity remained constant corresponding to the magnitude of positive pressure, whereas droplet volume increased with duration time up to the saturation volume. By controlling duration time over which positive pressure is applied, our system can dispense various droplet volumes at a desired droplet velocity.

\section{Flow resistance of the outlet}

Results of the previous experiment indicate that flow resistance (caused by liquid viscosity) at the outlet of our dispenser affects the volume and velocity of the droplet:

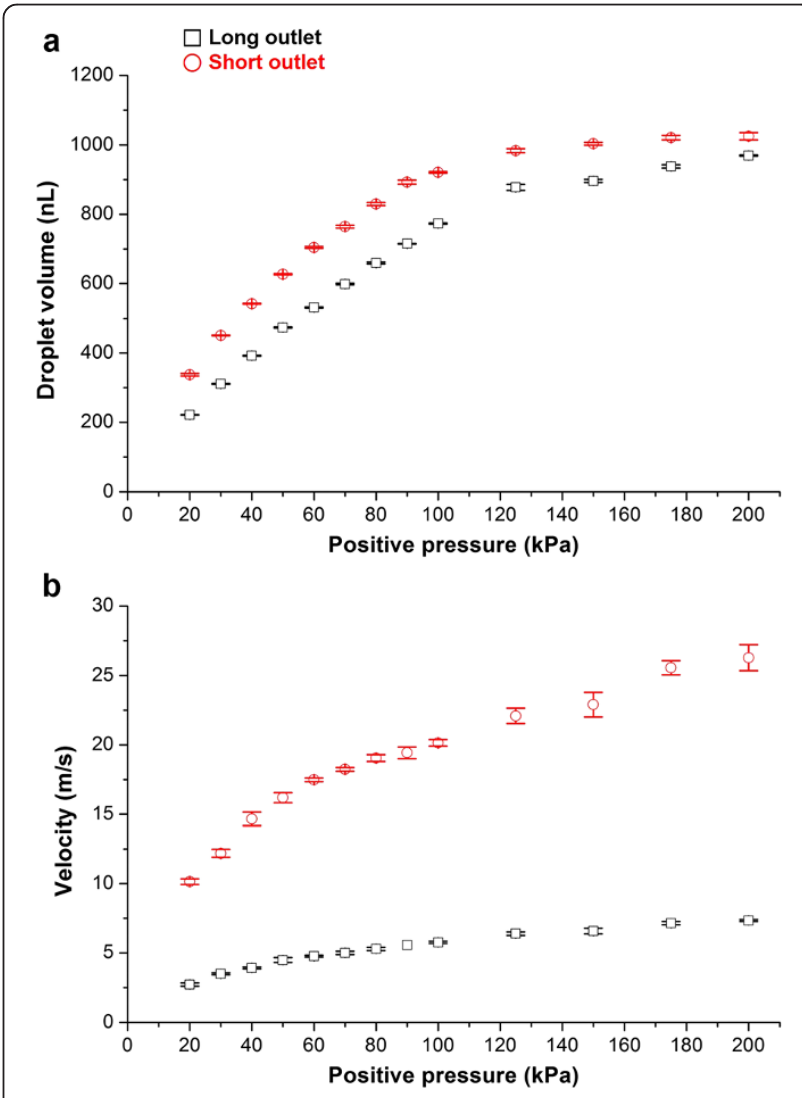

Figure 7 Comparison of two different outlet lengths: plots of (a) droplet volume and (b) velocity vs. positive pressure. 
small OW decreased droplet volume and velocity due to induced high flow resistance which causes large energy loss. Flow resistance of the outlet depends on its crosssection area and length. To confirm the effect of the flow resistance at the outlet under constant cross-section area, we measured the volume and velocity of droplets using dispensers which had 890- $\mu \mathrm{m}$ (long) and 75- $\mu \mathrm{m}$ (short) outlet length (CD $3 \mathrm{~mm}$ and OW $100 \mu \mathrm{m}$ ).

Figure 7 shows the measured droplet volume and velocity for two different outlet lengths with various positive pressures from $20 \mathrm{kPa}$ to $200 \mathrm{kPa}$. The short outlet shows that droplet volume at the same positive pressure increased by $6 \%$ to $50 \%$ compared with the long outlet (Figure $7(\mathrm{a})$ ), but droplet velocity at the same positive pressure greatly increased by $260 \%$ to $330 \%$ (Figure 7 (b)). The short outlet greatly increased droplet velocity due to reduction of flow resistance at the outlet.

\section{Conclusions}

We developed a pneumatic dispensing system to control the velocity of nanoliter droplets with small variation of their volume. The ability of the dispensing system to control droplet velocities was assessed under various design parameters and operating conditions. Droplet volume saturated because the maximum deflected volume of the membrane is limited by the chamber size. Droplet velocity increased depending on applied positive pressure after volume saturation. The velocity of droplets was affected mainly by the magnitude of positive pressure regardless of the duration time over which it was applied. The range of droplet velocities was strongly affected by the flow resistance at the outlet when the cross-section area of the outlet was held constant. The velocity of droplets ejected by our dispensing system can be easily controlled at a desired droplet volume by selecting design parameters and operating conditions that correspond to a specific patterning condition. For successful achievement of a patterning process, this dispensing system will provide a reliable performance within an optimized condition stably to deposit droplets onto accurate locations.

\section{Competing interests}

The authors declare that they have no competing interests.

\section{Authors' contributions}

$S L$ carried out the experiments, analyzed the experimental result, and drafted the manuscript. IHC performed the fabrication of micro device and conducted experiments. YKK and JK carried out experimental measurements and analysis. All authors read and approved the final manuscript.

\section{Acknowledgement}

This work was supported by the National Research Foundation of Korea (NRF) grant founded by the Korea government (MSIP) (No. 2011-0030075).

Received: 19 March 2014 Accepted: 19 May 2014

Published online: 12 July 2014

\section{References}

1. de Gans BJ, Duineveld PC, Schubert US (2004) Inkjet printing of polymers: state of the art and future developments. Advanced Materials 16(3):203-213. doi:10.1002/adma.200300385

2. Hon KKB, Li L, Hutchings IM (2008) Direct writing technology-advances and developments. CIRP Annals - Manufacturing Technology 57(2):601-620. doi:10.1016/j.cirp.2008.09.006

3. Singh $M$, Haverinen HM, Dhagat $P$, Jabbour GE (2010) Inkjet printingprocess and its applications. Advanced Materials 22(6):673-685. doi:10.1002/ adma.200901141

4. de Gans B-J, Schubert US (2003) Inkjet printing of polymer micro-arrays and libraries: instrumentation, requirements, and perspectives. Macromolecular Rapid Communications 24(11):659-666. doi:10.1002/ marc.200350010

5. Cheng S, Chandra S (2003) A pneumatic droplet-on-demand generator. Exp Fluids 34(6):755-762. doi:10.1007/s00348-003-0629-6

6. Sirringhaus $H$, Kawase $T$, Friend $\mathrm{RH}$, Shimoda $\mathrm{T}$, Inbasekaran $\mathrm{M}, \mathrm{Wu}$ W Woo EP (2000) High-resolution inkjet printing of all-polymer transistor circuits. Science 290(5499):2123-2126. doi:10.1126/science.290.5499.2123

7. Miettinen J, Pekkanen V, Kaija K, Mansikkamäki P, Mäntysalo J, Mäntysalo M, Niittynen J, Pekkanen J, Saviauk T, Rönkkä R (2008) Inkjet printed systemin-package design and manufacturing Microelectronics Journal 39(12):1740-1750. doi:10.1016/j.mejo.2008.02.014

8. Chin-Tai C, Kuo-Hua W, Chun-Fu L, Fanny S (2010) An inkjet printed stripe-type color filter of liquid crystal display. Journal of Micromechanics and Microengineering 20(5):055004

9. Attinger D, Zhao Z, Poulikakos D (2000) An experimental study of molten microdroplet surface deposition and solidification: transient behavior and wetting angle dynamics. Journal of Heat Transfer 122(3):544-556. doi:10.1115/1.1287587

10. Calvert $P$ (2001) Inkjet printing for materials and devices. Chemistry of Materials 13(10):3299-3305. doi:10.1021/cm0101632

11. Kwak D, Lim JA, Kang B, Lee WH, Cho K (2013) Self-organization of inkjet-printed organic semiconductor films prepared in inkjet-etched microwells. Advanced Functional Materials 23(42):5224-5231. doi:10.1002/adfm.201300936

12. Boland T, Xu T, Damon B, Cui X (2006) Application of inkjet printing to tissue engineering. Biotechnology Journal 1(9):910-917. doi:10.1002/ biot.200600081

13. Arrabito G, Pignataro B (2010) inkjet printing methodologies for drug screening. Analytical Chemistry 82(8):3104-3107. doi:10.1021/ac100169w

14. Hook AL, Anderson DG, Langer R, Williams P, Davies MC, Alexander MR (2010) High throughput methods applied in biomaterial development and discovery. Biomaterials 31(2):187-198. doi:10.1016/j.biomaterials.2009.09.037

15. Kang B, Min H, Seo U, Lee J, Park N, Cho K, Lee HS (2013) Directly drawn organic transistors by capillary pen: a new facile patterning method using capillary action for soluble organic materials. Advanced Materials 25 (30):4117-4122. doi:10.1002/adma.201300006

16. Derby B (2010) Inkjet printing of functional and structural materials: fluid property requirements, feature, stability, and resolution. Annual Review of Materials Research 40(1):395-414. doi:10.1146/annurev-matsci-070909104502

17. Sirringhaus $H$, Shimoda $T$ (2003) Inkjet printing of functional materials. MRS Bulletin 28(11):802-806

18. Brown PS, Berson A, Talbot EL, Wood TJ, Schofield WCE, Bain CD, Badyal JPS (2011) Impact of picoliter droplets on superhydrophobic surfaces with ultralow spreading ratios. Langmuir: the ACS journal of surfaces and colloids 27(22):13897-13903. doi:10.1021/la203329n

19. Jang D, Kim D, Moon J (2009) Influence of fluid physical properties on ink-jet printability. Langmuir: the ACS journal of surfaces and colloids 25(5):2629-2635. doi:10.1021/la900059m

20. Tekin E, Smith PJ, Schubert US (2008) Inkjet printing as a deposition and patterning tool for polymers and inorganic particles. Soft Matter 4(4):703-713. doi:10.1039/B711984D

21. Wijshoff $H$ (2010) The dynamics of the piezo inkjet printhead operation. Physics Reports 491(4-5):77-177, doi:10.1016/j.physrep.2010.03.003

22. Sun J, Ng J, Fuh Y, Wong Y, Loh H, Xu Q (2009) Comparison of microdispensing performance between micro-valve and piezoelectric printhead. Microsyst Technol 15(9):1437-1448. doi:10.1007/s00542-009-0905-3

23. Amirzadeh Goghari A, Chandra S (2008) Producing droplets smaller than the nozzle diameter by using a pneumatic drop-on-demand droplet generator. Exp Fluids 44(1):105-114. doi:10.1007/s00348-007-0378-z 
24. Lee S, Kim J (2010) Development and characterization of a cartridge-type pneumatic dispenser with an integrated backflow stopper. Journal of Micromechanics and Microengineering 20(1). doi:10.1088/0960-1317/20/1/015011

25. Lide DR (2005) CRC Handbook of Chemistry and Physics (86th ed.). Taylor \& Francis Group Boca Raton, FL

26. Beebe DJ, Mensing GA, Walker GM (2002) Physics and applications of microfluidics in biology. Annual Review of Biomedical Engineering 4(1):261-286. doi:10.1146/annurev.bioeng.4.112601.125916

doi:10.1186/s40486-014-0005-8

Cite this article as: Lee et al:: Velocity control of nanoliter droplets using a pneumatic dispensing system. Micro and Nano Systems Letters 2014 2:5.

Submit your manuscript to a SpringerOpen ${ }^{\circ}$ journal and benefit from:

- Convenient online submission

- Rigorous peer review

- Immediate publication on acceptance

- Open access: articles freely available online

- High visibility within the field

- Retaining the copyright to your article

Submit your next manuscript at $\gg$ springeropen.com 